J Venom Anim Toxins incl Trop Dis, 2019 25: e20190001

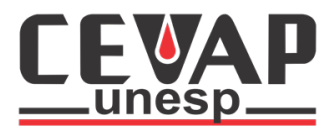

\author{
The Journal of Venomous Animals and \\ Toxins including Tropical Diseases \\ ISSN 1678-9199 \\ Journal homepage www.jvat.org
}

\title{
Using prodigiosin against some gram-positive and gram-negative bacteria and Trypanosoma cruzi
}

\author{
Rocío Herráez ${ }^{1}$, Anna Mur ${ }^{1}$, Alexandra Merlos ${ }^{1}$, Miguel Viñas ${ }^{1}$, Teresa Vinuesa ${ }^{1, *}$ (D) \\ ${ }^{1}$ Department of Pathology and Experimental Therapeutics, Medical School and IDIBELL, Campus Bellvitge, University of Barcelona, \\ L'Hospitalet de Llobregat, Barcelona, Spain
}

\section{Article Info \\ Keywords:}

Trypanosoma cruzi

Chagas disease

Prodigiosin

Atomic force microscopy

\begin{abstract}
Background: This work aimed to explore the action of natural prodigiosin on both bacterial organisms and Trypanosoma cruzi cells.
\end{abstract}

Methods: Natural prodigiosin pigment was extracted and purified from cultures of Serratia marcescens. Two media, peanut broth and peptone glycerol broth, both recommended in the literature for prodigiosin production, were compared. The prodigiosin obtained was employed to explore its antimicrobial properties against both bacteria and Trypanosoma cruzi cells.

Results: Peanut broth yielded four times more prodigiosin. The prodigiosin showed remarkable activity (minimal inhibitory concentrations in the range of $2-8 \mu \mathrm{M}$ for bacteria and half maximal inhibitory concentration of $0.6 \mu \mathrm{M}$ for Trypanosoma cruzi). In fact, the prodigiosin concentration required to inhibit parasite growth was as low as 0.25 $\mathrm{mg} / \mathrm{l}$ versus $4.9 \mathrm{mg} / \mathrm{l}$ of benznidazole required. Furthermore, atomic force microscopy revealed marked morphological alterations in treated epimastigote forms, although no pore-formation activity was detected in protein-free environments.

Conclusions: This work demonstrates the potential usefulness of prodigiosin against some gram-positive and gram-negative bacteria and Trypanosoma cruzi although further studies must be done in order to assess its value as a candidate molecule.

\footnotetext{
* Correspondence: 


\section{Background}

Prodigiosin is a linear tripyrrol produced as a secondary metabolite by different bacteria, including Serratia marcescens, Vibrio spp., Pseudomonas spp. and Streptomyces spp., among others [1]. It is a highly stable pigment, and a member of the alkaloid family called prodigionines, presenting a blood-red color at acidic $\mathrm{pH}$ values. Its synthesis is closely related to amino-acid metabolism [2,3]. Although its biological role in the producer microorganisms has not yet been elucidated, it has demonstrated a high electron transport capacity. Prodigiosin is capable of uncoupling $\mathrm{H}^{+} / \mathrm{Cl}^{-}$transporters, since it can bind to and transport chloride; subsequently, it modulates the $\mathrm{pH}$ of the bacterial cell [4]. Notwithstanding the scarce knowledge of its mechanism of action, prodigiosin appears as a pluripotent molecule, with various health-related properties; the most important being: an anticancer agent $[5,6]$, an immunosuppressant, an antiprotozoal and an antibacterial agent, while also offering protection against UV $[7,8,9]$. Different studies have shown that prodigiosin inhibits the growth of a broad spectrum of gram-positive (Staphylococcus spp., Bacillus spp., etc.) as well as gram-negative (Escherichia coli, Salmonella enterica, etc.) bacteria [10]. Its capacity to inhibit the growth of eukaryotic microorganisms such as fungi (Candida albicans) and protozoa (Plasmodium spp. and Trypanosoma spp.) has also been reported [11,12,13].

The first report of the in vitro effect of prodigiosin on Trypanosoma cruzi dates from more than 65 years ago [14] when concentrations as low as $3 \mu \mathrm{M}$ showed the capacity to completely inhibit the parasite. Azambuja et al. [15] reported interactions between Trypanosoma cruzi and a producer of prodigiosin, namely Serratia marcescens. Moreover, those authors succeeded in demonstrating that the loss of trypanosomal activity of Serratia marcescens DB11 was concomitant with the loss of capacity to produce prodigiosin, thereby indicating that the tripyrrolic pigment is a significant contributor to antitrypanosomal activity. Furthermore, the eventual usefulness of prodigiosin in the treatment of Chagas disease has been explored. Genes et al. [16] indicated that the activity of prodigiosin may mediate an apoptotic phenomenon in Trypanosoma cruzi by induction of mitochondrial dysfunctions. Finally, prodigiosin and another bacterial pigment (violacein from Chromobacterium violaceum), as well as their combinations with silver and gold nanoparticles, have been studied for their in vitro inhibition of the growth of Plasmodium falciparum and Trypanosoma brucei $[17,18]$. Prodigiosin was found to be more effective than violacein at inhibiting both of these parasites; moreover, combinations of prodigiosin and metal nanoparticles resulted in increased parasiticidal activity, while toxicity for mammalian cells remained stable.

Nevertheless, the antimicrobial properties of prodigiosin have often been questioned, particularly because of the high concentrations required for it to be effective, as these exceed the levels causing toxicity in mammalian cells. For this reason, it has been studied in greater depth for its use in anticancer and immunosuppressive therapy, than as an agent to fight infectious agents $[19,20]$.

The interest in prodigiosin as a drug is clearly demonstrated by the number of reports in the literature from the different fields concerned, as well as by the explorations of its complexation and encapsulation, for both drug-delivery and to enhance its activity [21].

Our group has acquired considerable expertise in dealing with not only the physiology and properties of prodigiosin but also its production by Serratia marcescens. We are specifically interested in the antimicrobial activity of prodigiosin, including its antitrypanosomal activity $[6,15,16]$.

In the present study, we focused our efforts on purifying crude extracts of bacterial origin and determining their activity against the parasite, in the hope of lowering the concentration needed to inhibit growth of the parasite, as well as decreasing its toxicity to mammalian cells.

We compared two methods of prodigiosin production by evaluating the effect of peanut broth and peptone/glycerol broth on production of the pigment [22]. We also considered extraction methods by using organic solvents and setting up a purification system using an X-5 resin-affinity chromatography column [23] to purify the molecule in order to test whether such a procedure can increase its effectiveness at reducing toxicity.

Therefore, the present work aimed to explore and compare the action of the prodigiosin obtained on both bacterial organisms and Trypanosoma cruzi cells. In the case of the parasite, prodigiosin action was studied by determination of its anti-epimastigote activity, as well as by visualization of injuries and measurements of cell damage by atomic force microscopy (AFM). Furthermore, we have investigated the eventual alterations induced in membranes by the pigment through electrophysiological measurements of the conductance capacity in black lipid bilayers and by searching for channel forming activity.

\section{Methods}

\section{Bacterial strains and cultures}

The following bacterial strains were used to measure susceptibility: Staphylococcus aureus ATCC 29213, and ATCC 700698, Enterococcus faecalis ATCC 29212, Escherichia coli ATCC 25922, Pseudomonas aeruginosa ATCC 27853, plus one isolate of Bacillus subtilis and one of B. pumilus, from our own collection. They were thawed and maintained by daily passage in Columbia Blood Agar until the experiments were performed. Serratia marcescens 2170 was used as the prodigiosin producer.

\section{Parasite culture}

For Trypanosoma cruziCL-B5 maintenance, epimastigotes were cultured axenically at $28^{\circ} \mathrm{C}$ in liver infusion-tryptose medium (LIT; DifcoTM BD, MD, USA; Conda Pronadisa, Madrid, Spain) supplemented with $10 \%$ heat-inactivated fetal bovine serum (FBS; Gibco, Life Technologies, AU, NZ) plus antibiotics $(100 \mu \mathrm{g}$ streptomycin sulfate/ml and $100 \mathrm{U}$ penicillin G sodium salt/ml, 
SP; Sigma Aldrich, CA, USA). The CL-B5 strain was maintained in exponential growth by weekly passages [24,25].

\section{Prodigiosin preparation}

For prodigiosin production, Serratia marcescens 2170 was cultured overnight in peptone-glycerol medium under orbital shaking $(250 \mathrm{rpm})$ at $30^{\circ} \mathrm{C}$. Then, $75 \mathrm{ml}$ of bacterial culture was spread onto peptone-glycerol agar and incubated for 48 hours at $28^{\circ} \mathrm{C}$. After incubation, the bacteria were harvested by scraping the plate surfaces, suspended in methanol: $\mathrm{HCl}(24: 1 \mathrm{v} / \mathrm{v})$, and centrifuged $(8,000 \mathrm{xg}$ for $15 \mathrm{~min})$ to eliminate debris. The pigment was then collected and concentrated in a rotary evaporator. These prodigiosin preparations were denominated PG 1 .

In addition, after overnight culture in peptone-glycerol medium, Serratia marcescens 2170 was transferred to 11 Erlenmeyer flasks $(2.5 \mathrm{ml} / \mathrm{l})$ and incubated for $72 \mathrm{~h}$ at $28^{\circ} \mathrm{C}$ in 250 $\mathrm{ml}$ of peanut medium at $250 \mathrm{rpm}$. The peanut medium consisted of $2 \%$ crushed peanut seeds in distilled water, autoclaved at $121^{\circ} \mathrm{C}$ for $20 \mathrm{~min}$. The bacteria were centrifuged $(8,000 \mathrm{x} \mathrm{g}$ for 15 $\mathrm{min})$, washed twice and finally the pigment was extracted using a mixture of methanol: $\mathrm{HCl}(24: 1 \mathrm{v} / \mathrm{v})$, and shaken overnight at $250 \mathrm{rpm}$. After centrifugation to eliminate debris, the pigment was collected and concentrated in a rotary evaporator. These prodigiosin preparations were called PG 2 .

In another batch of production, the supernatants from both of the previous media were collected and purified by the technique described by Wang et al. [23], with some modifications, as we did not use raw material but rather previously extracted prodigiosin.

After the X-5 resin had been hydrated (in MilliQ water), the column was packed and washed successively with water to remove impurities. A layer of sand was added above the packed column, and then the pigment solution was poured into the column. Elution was carried out with methanol and colored fractions were collected.

Purified prodigiosin produced from the peptone-glycerol medium was denominated PG 3; and the purified prodigiosin produced from the peanut medium was called PG 4 .

The purified prodigiosin was evaluated using: spectrophotometric analysis with absorbance determined in the range $450-700 \mathrm{~nm}$; thin-layer chromatography (TLC); and electrospray ionization mass spectrometry ESI-MS. The prodigiosin concentration was calculated using the Beer-Lambert law, considering a value for the molar extinction coefficient of $110,000 \mathrm{M}^{-1} \cdot \mathrm{cm}^{-1}$ and using the maximum $535 \mathrm{~nm}$ absorbance value.

\section{Antibacterial effect}

Values of minimal inhibitory concentration (MIC) were determined by the broth microdilution method and interpreted according to the EUCAST guidelines. Briefly, an isolate of each bacterial strain was grown at $37^{\circ} \mathrm{C}$ and $250 \mathrm{rpm}$ overnight in 20 $\mathrm{mL}$ of Tryptic Soy Broth medium. Next, $800 \mu \mathrm{L}$ of the culture was utilized to inoculate $20 \mathrm{~m} \mathrm{~L}$ of Mueller-Hinton medium. After $2 \mathrm{~h}$ at $37^{\circ} \mathrm{C}$ and $250 \mathrm{rpm}$, bacterial inocula were adjusted with Ringer up to an optical density at $625 \mathrm{~nm}$ from 0.08 to 0.1
(0.5 McFarland; $10^{7}-10^{8} \mathrm{ufc} / \mathrm{m} \mathrm{L}$ ). Five (5) $\mu \mathrm{L}$ of each bacterial culture was added to a 96 -well microplate previously filled with serial dilutions of prodigiosin. The plates were incubated at $37^{\circ} \mathrm{C}$ for $24 \mathrm{~h}$. The MIC was determined macroscopically, by assessing turbidity with the aid of a mirrored viewbox, scoring the lowest concentration well without turbidity. Growth and control wells were also included. Each concentration was run in triplicate and the experiments were repeated three times independently.

\section{Epimastigote susceptibility}

Seven-day-old culture epimastigotes $\left(2.5 \times 10^{5} / \mathrm{ml}\right)$ were incubated in 96-well microplates (WWR Int LLC) with serial dilutions of natural prodigiosins for 72 hours at $28^{\circ} \mathrm{C}$. Growth and control wells with only the parasite and the reference drug benznidazole (ELEA, Buenos Aires, Argentina), respectively, were also included in the assay. Each concentration was run in triplicate and the experiments were repeated three times independently. Parasite growth was checked by cell counting in a Neubauer chamber.

The efficacy of the compounds was calculated by determining the epimastigote percentage growth, as follows:

$$
[(C D 3 t-C D 0) /(C D 3 c-C D 0)] \times 100
$$

where $\mathrm{CD} 3 \mathrm{t}$ is the epimastigote concentration of treated parasites at day 3; $\mathrm{CD} 0$ is the epimastigote concentration at day 0 ; and $\mathrm{CD} 3 \mathrm{c}$ is the epimastigote concentration of untreated parasites at day 3 [26].

The $\mathrm{IC}_{50}$ values (half maximal inhibitory concentration) were calculated using GraphPad Prism software.

\section{Atomic Force Microscopy (AFM)}

Since the activity of prodigiosin against microorganisms is attributed to plasma-membrane damage [27], we explored the effect of prodigiosin on epimastigote forms of Trypanosoma cruzi using AFM, by comparison between treated and untreated parasites. These alterations were also compared with those induced by the benznidazole treatments.

Epimastigote forms of T. cruzi (CLB strain) cultivated in LIT medium, after exposure to prodigiosin $1.26 \mu \mathrm{M}$ and benznidazole $48 \mu \mathrm{M}$ for $72 \mathrm{~h}$, were visualized using an XE-70 atomic force microscope (Park Systems, Korea). We used these two concentrations [ 2 times $50 \%$ inhibitory concentration value $\left.\left(\mathrm{IC}_{50}\right)\right]$ since, although the parasite loses the capacity to grow, it is not completely destroyed and it is still feasible for visualizing organism surfaces and injuries. The parasites were collected by centrifugation, washed in phosphate buffered saline (PBS) $0.1 \mathrm{M}$, fixed with glutaraldehyde $2.5 \%$ in PBS $0.1 \mathrm{M}$ for 1 hour, washed twice with $5 \mathrm{ml}$ of MilliQ water and placed onto a MICA surface, allowed to dry at room temperature and then imaged in air using the AFM equipment. All images were collected in non-contact mode using pyramidal-shaped silicon cantilevers with a spring constant of $\pm 40 \mathrm{~N} / \mathrm{m}$ and a resonance frequency of $\pm 300 \mathrm{kHz}$, with the upper side coated with aluminum to enhance the laser 
beam reflectivity. The data acquired during the surface scanning were converted into images of topography and amplitude, and analyzed using XEP and XEI software (Park Systems, Korea). In the topography images, it was possible to observe the shape, structure, and surface of individuals. Furthermore, amplitude images enabled visualization of fine surface details. The AFM images were acquired with a scan size of $20 \mu \mathrm{m}^{2}$ at a scan rate of $0.3-0.6 \mathrm{~Hz}$. AFM was also employed to measure the surface roughness of the treated and untreated individuals. The roughness average $(\mathrm{Ra})$, defined as the average distance from the roughness profile to the center plane of the profile, was calculated from the topography images obtained in these experiments.

\section{Statistical analysis}

Both height and surface nano-roughness averages of the parasite cells after treatment with prodigiosin and benznidazole were compared and statistically analyzed by one-way ANOVA and Tukey's test. Differences were considered significant when $\mathrm{p}<0.05$.

\section{Lipid bilayer experiments}

The eventual channel-forming activity was explored via preparation of artificial lipid bilayers and the subsequent addition of prodigiosin. The black lipid bilayer experiments were carried out via the method reported by Benz et al. [28]. Membranes were formed by forming a $0.8 \mathrm{~mm}^{2}$ hole in a divider separating two compartments of the Teflon chamber containing $5 \mathrm{~mL}$ each of a bathing solution of salt, with $1 \%$ (w/v) lipid diphytanoyl phosphatidylcholine solution in n-decane (Avanti Polar Lipids). All salts (analytical grade) were obtained from Sigma Aldrich, Spain. Voltages were applied across the membrane formed via $\mathrm{Ag} / \mathrm{AgCl}$ electrodes connected by a salt bridge, and the resultant current was boosted by a factor of $10^{9}-10^{10}$ by a current amplifier, and recorded on a Rikadenki strip chart recorder. The temperature was maintained at $20^{\circ} \mathrm{C}$ throughout.

In order to evaluate channel-forming activity, increasing volumes of PG 3, starting at $1 \mu \mathrm{l}$ and arriving at a final volume of $50 \mu \mathrm{l}$, were added to the aqueous phase of both sides of the bilayer, until a final concentration of $0.44 \mu \mathrm{M}$ was reached on each side.

For PG 4, after the addition of increasing volumes, starting at $1 \mu \mathrm{l}$ and arriving at a final volume of $50 \mu \mathrm{l}$, we achieved a final concentration of $0.94 \mu \mathrm{M}$ on each side.

\section{Results}

\section{Prodigiosin production}

Both methods employed to produce prodigiosin to perform the further experiments yielded apparently clean pigment solutions that were relatively pure and adequate for the experiments. However, the pigment yield when bacteria were cultured in peanut broth was much higher, as shown in Table 1. In all cases, purity was determined and proven to be acceptable, as can be concluded from Figure 1, which shows absorption spectra with the typical peak at $535 \mathrm{~nm}$ and a shoulder at $515 \mathrm{~nm}$; while no other peaks or disturbances were observed. Mass spectrometry confirmed the results (data not shown).

Table 1. Preparation of prodigiosin for experimental determination of antitrypanosomal and antibacterial action.

\begin{tabular}{ccccc}
\hline $\begin{array}{c}\text { Prodigiosin } \\
\text { preparation code }\end{array}$ & Medium & Extraction & Purification & $\begin{array}{c}\text { Final prodigiosin } \\
\text { concentration }(\boldsymbol{\mu} \text { M) }\end{array}$ \\
\hline PG 1 & Peptone-glycerol & Methanol: $\mathrm{HCl}(24: 1)$ & $\mathrm{ND}$ & 42 \\
PG 2 & Peanut & Methanol: $\mathrm{HCl}(24: 1)$ & $\mathrm{ND}$ & 160 \\
PG 3 & Peptone-glycerol & Methanol: $\mathrm{HCl}(24: 1)$ & X-5 Resin & 44 \\
PG 4 & Peanut & Methanol: $\mathrm{HCl} \mathrm{(24:1)}$ & X-5 Resin & 177 \\
\hline
\end{tabular}

ND: not done

\section{Antibacterial effect}

Purified prodigiosin preparations (PG 3 and PG 4) displayed remarkable antimicrobial activity, as can be concluded from the
MIC values (Table 2) against all the bacterial strains tested, which represented the major groups of pathogenic microorganisms except Mycobacterium.

Table 2. MIC of prodigiosin against the different bacterial strains tested.

\begin{tabular}{lcc}
\hline Bacterial strain & MIC $(\boldsymbol{\mu M})$ \\
\hline S. aureus ATCC 29213 & 4 \\
S. aureus ATCC 700698 & 4 \\
B. subtilis & 2 & 2 \\
B. pumilus & 2 \\
E. faecalis ATCC 29212 & 2 \\
E. coli ATCC 25922 & 8 \\
P. aeruginosa ATCC 27853 & 2 \\
\hline
\end{tabular}




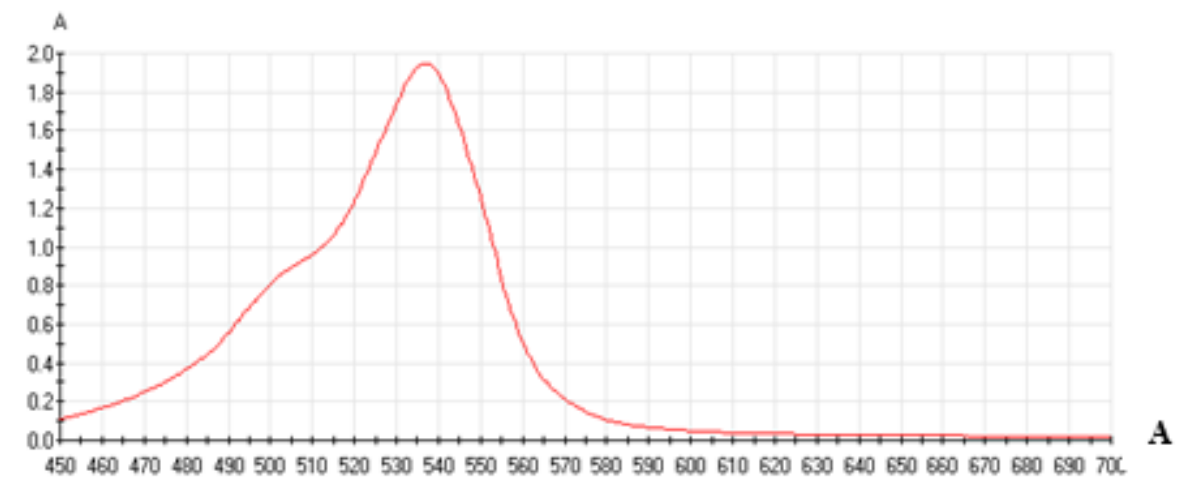

$\mathrm{rm}$

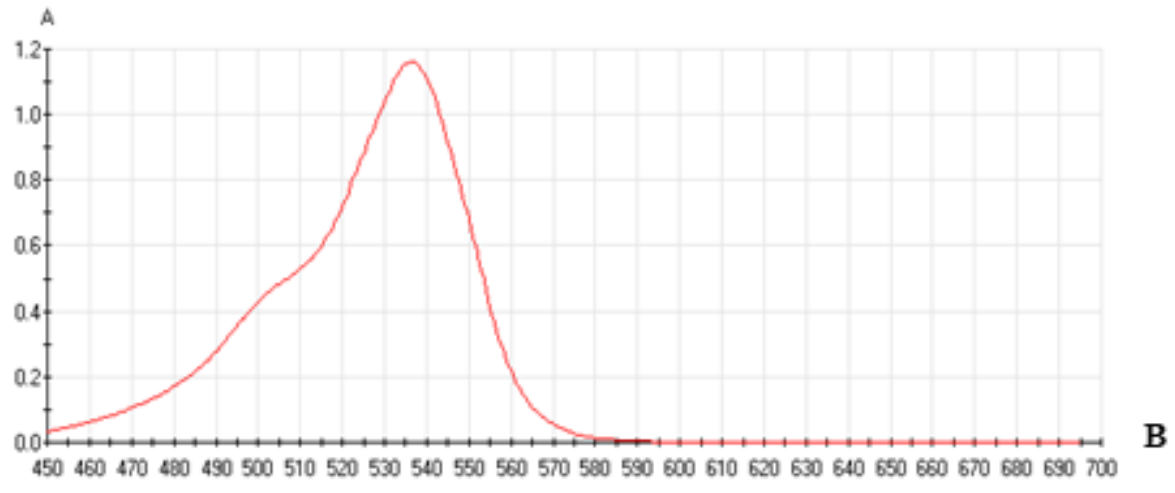

$\mathrm{rm}$

Figure 1. Visible adsorption spectra (range: $450-700 \mathrm{~nm}$ ) of purified prodigiosin obtained from peptone-glycerol medium (A) and peanut broth (B).

\section{Epimastigote susceptibility}

Similarly, the preparations were highly active against parasites in the epimastigote state (Fig. 2). Table 3 shows values of antiepimastigote activity $\left(\mathrm{IC}_{50}\right)$. Activity of both chromatographically purified prodigiosin preparations gave lower $\mathrm{IC}_{50}$ values than those in a native state (just extracted, without purification). The value for the drug currently utilized to treat Chagas disease (benznidazole) is also reported, to facilitate comparison.

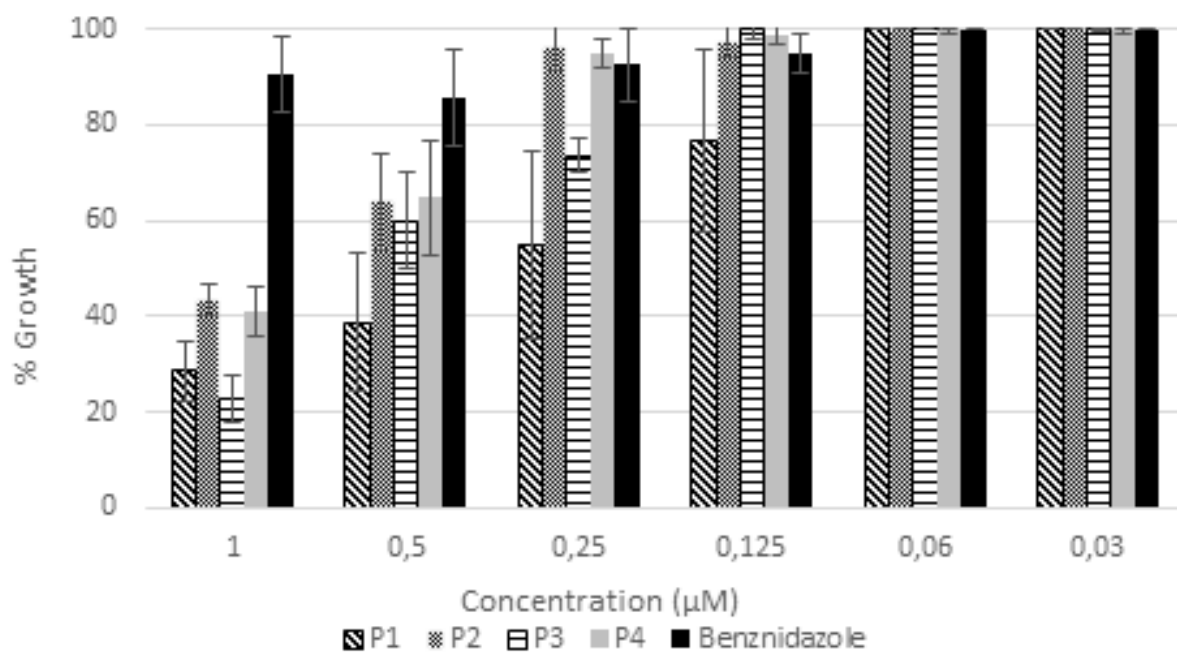

Figure 2. Biological activity of benznidazole and prodigiosin preparations against T. cruzi epimastigotes of the CL-B5 strain expressed as percentage of growth with corresponding standard deviation. 
Table 3. Biological activity of natural, extracted and purified prodigiosin preparations against $T$. cruzi epimastigotes (CL strain, clone B5).

\begin{tabular}{cc}
\hline Anti-epimastigote activity & IC $_{\mathbf{5 0}}(\boldsymbol{\mu M}) \pm \mathbf{S D}$ \\
\hline PG 1 & $0.80 \pm 0.25$ \\
PG 2 & $0.78 \pm 0.08$ \\
PG 3 & $0.60 \pm 0.1$ \\
PG 4 & $0.60 \pm 0.40$ \\
Benznidazole & $18.9 \pm 7.06$ \\
\hline
\end{tabular}

$1 C_{50} 50 \%$ inhibitory concentration

\section{AFM}

Epimastigote forms of T. cruzi treated with prodigiosin (PG 3) showed severe morphological alterations with loss of membrane integrity, as can be concluded by observing the corresponding images (Figure 3). Moreover, several parameters were affected after treatment, such as average height of the parasite cell (Figure $4 \mathrm{a}$ ) and surface nano-roughness (Figure $4 \mathrm{~b}$ ).

\section{Lipid bilayer experiments}

When the extracts of prodigiosin (PG 3 and PG 4) were added to bathing solutions separated by black lipid bilayer membranes, no significant increases in conductance were observed. This indicates that, at least in lipid bilayers constructed in our laboratory, the incorporation of prodigiosin into the bilayer, when it happened, did not lead to formation of transmembrane channels at any of the concentrations assayed in our experiments. We conclude that there was no pore-forming activity of prodigiosin in protein-free environments.

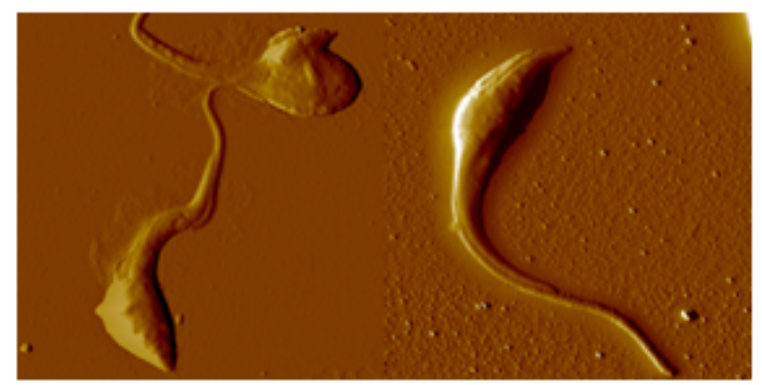

A

\section{Discussion}

Prodigiosin production was compared between the two media used (peptone-glycerol medium and peanut broth), revealing that peanut medium yields much more pigment (up to fourfold more) as shown in Table 1. Thus, when the goal is to produce natural prodigiosin, peanut broth would be efficacious. Our results confirm (and optimize) those reported by Giri et al. [22].

All four prodigiosin preparations obtained from $S$. marcescens 2170 cultures exhibited a marked antibacterial effect, similar to that already reported by other authors [27]. We obtained evidence that biological features of the prodigiosin obtained in our laboratory were maintained during the different extraction and purification procedures. When measuring antitrypanosomal action, there were no differences due to the media used in the production step (peptone-glycerol medium and peanut broth): the antitrypanosomal action was identical. Moreover, purification using X-5 resin increased antitrypanosomal effectiveness. This may be attributed to a higher purity level and the subsequent

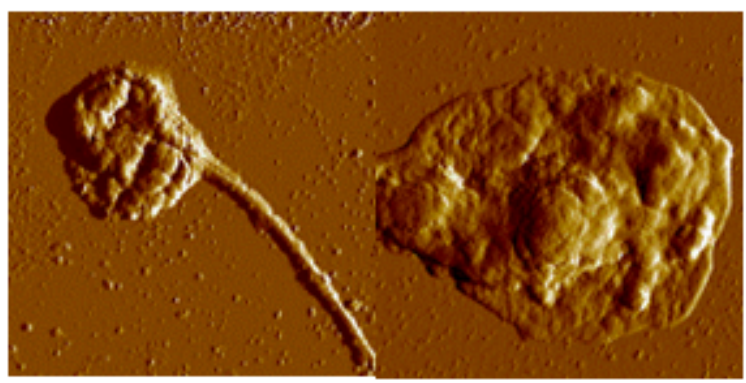

B

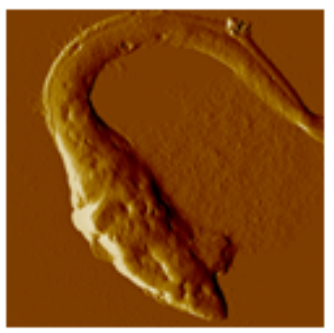

C

Figure 3. AFM amplitude images obtained at a $20 \mu \mathrm{m}^{2}$ scan size, of: (A) untreated T. cruzi epimastigotes; (B) T. cruzi epimastigotes after $72 \mathrm{~h}$ of treatment with PG 3, $1.26 \mu \mathrm{M}$; (C) T. cruzi epimastigotes after $72 \mathrm{~h}$ of treatment with benznidazole, $48 \mu \mathrm{M}$. 


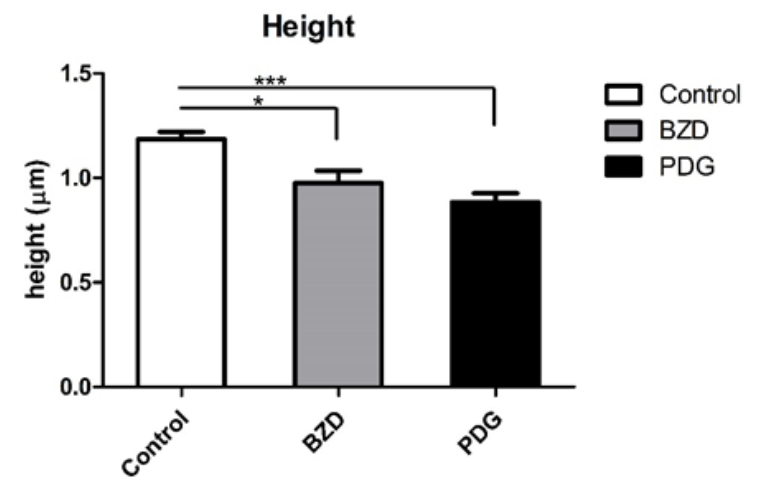

A

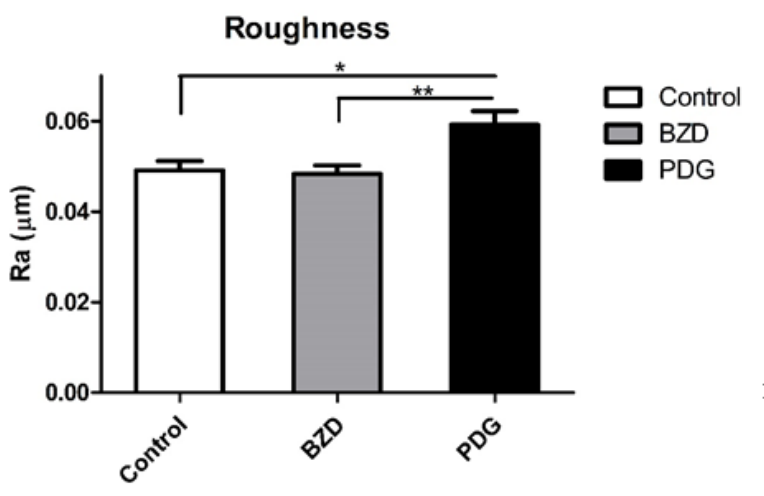

Figure 4. (A) Average height in micrometers, for the different formulations tested and surface scans. Bars represent the standard deviation of the mean of at least 30 measurements. *, $\mathrm{P}<0.05$; ***, $\mathrm{P}<0.001$. (B) Surface roughness $(\mathrm{Ra})$ in micrometers, for the different formulations tested and surface scans. Bars represent the standard deviation of the mean of at least 30 measurements. *, $\mathrm{P}<0.05 ; * *, \mathrm{P}<0.01$.

absence of interfering remains of bacteria and debris. In fact, when comparing the data, we can conclude that the benznidazole concentrations required to inhibit parasite growth are clearly higher than those needed with prodigiosin $(4.9 \mathrm{mg} / \mathrm{l}$ and 0.25 $\mathrm{mg} / \mathrm{l}$, respectively).

When the capacity of prodigiosin to form channels was explored, our measurements did not indicate any insertion into the artificial membranes created. That is to say, no increases in conductance were detected. In principle, the addition of an ion transporter into a membrane should result in discrete increases of conductance; the lack of positive results may be a consequence of an incapacity of the molecule to become inserted into the bilayer. It has been shown that prodigiosin is a molecule that resides in bacterial membranes [29], while its antimicrobial action has been attributed to membrane injuries [27], which is apparently inconsistent with its failure to modify the membrane conductance observed herein. Nevertheless, artificial black lipid bilayers, despite acting electrophysiologically as cell membranes, are formed only by lipids and we cannot rule out the possibility that insertion of the prodigiosin, and its activity as an ion transporter, may require the cooperation of some membrane protein or some special domains of lipids other than the ones used in building artificial bilayers.
The effect of prodigiosin against gram-positive and gramnegative bacteria that we found is remarkable.

As to trypanocidal activity, the $\mathrm{IC}_{50}$ values of the purified prodigiosin preparations were more active than crude ones, probably due to the purity of their composition.

The action mechanism of prodigiosin as an antimicrobial agent is not fully established, although the acquired AFM images of treated epimastigotes showed remarkable changes on parasite cell surfaces as well as in height and roughness, suggesting a catastrophic scenario caused by the pigment. This is consistent and complements the results of Genes et al. [16] when reporting the activity of prodigiosin against $T$. cruzi, evidencing that the mode of action of prodigiosin involves the mitochondrial function. In fact, despite using different strains ( TcI and TCII) with different susceptibilities to Benznidazole, they found that classical inhibitors of respiratory function such as Rotenone, Thenoyltrifluoroacetone (TTFA), potassium cyanide (KCN) and antimycin $\mathrm{A}$, produce an effect on mitochondrial membrane potential similar to that of prodigiosin, but they were much less parasiticidal. Thus, it may be plausible that prodigiosin action takes place not only on mitochondria, but also in other cell compartments and mainly on plasma membrane.

In conclusion, the findings of the present study strongly indicate that prodigiosin exerts a potent action by killing $T$. cruzi in its epimastigote form. These findings suggest that the bacterial secondary metabolite could become a resource in fighting Chagas disease. Therefore, additional studies need to be performed to verify its safety in relation to mammalian cells and intracellular and trypomastigote forms of the parasite.

\section{Abbreviations}

AFM: Atomic force microscopy; BZD: Benznidazole; ESI-MS: Electrospray ionization mass spectrometry; FBS: Foetal bovine serum; $\mathrm{IC}_{50}: 50 \%$ inhibitory concentration; KCN: Potassium cyanide; LIT: Liver infusion-tryptose; MIC: Minimal inhibitory concentration; PBS: Phosphate buffered saline; PDG: Prodigiosin; PG 1: Prodigiosin 1; PG 2: Prodigiosin 2; PG 3: Prodigiosin 3; PG 4: Prodigiosin 4; Ra: Roughness average; TLC: Thin-layer chromatography; TTFA: Thenoyltrifluoroacetone.

\section{Availability of data and materials}

Available by request to the corresponding author.

\section{Funding}

This article is part of the European project BERENICE. BERENICE is a Collaborative Project that is funded under the European Community's 7th Framework Programme. Grant agreement number: HEALTH-30593. Moreover, this publication was supported in part by the Coordination for the Improvement of Higher Education Personnel (CAPES) through "Programa Editoração CAPES" and by the National Council for Scientific and Technological Development (CNPq) and (CAPES) through "Programa Editorial CNPq/CAPES". 


\section{Competing interests}

The authors declare that they have no competing interests.

\section{Authors' contributions}

AMS, RHM, TVA and MVC developed the experiments to obtain the prodigiosin; RHM performed the microbiology and lipid bilayer experiments; AMG performed the Atomic Force Microscopy work; TVA and MVC wrote the paper and are responsible for the design and general ideation of the work and also analyzed the data; All authors read and approved the final manuscript.

\section{Ethics approval and consent to participate}

Not applicable.

\section{Consent for publication}

Not applicable.

\section{References}

1. Williams RP. Biosynthesis of prodigiosin, a secondary metabolite of Serratia marcescens. Appl Microbiol. 1973;25(3):396-402.

2. Scott RH, Qadri SM, Williams RP. Role of L-proline in the biosynthesis of prodigiosin. Appl Environ Microbiol. 1976;32(4):561-6.

3. Leranoz AM, Fusté MC, Viñas M, Hull RA, Williams RP. Cloning and expression in Escherichia coli of a gene encoding proline oxidase of Serratia marcescens. Microbios. 1991;67(271):87-94.

4. Rius N, Solé M, Francia A, Lorén JG. Buffering capacity and H+ membrane conductance of gram-negative bacteria. FEMS Microbiol Lett. 1995;130(1):103-10.

5. Pérez-Tomás R, Montaner B, Llagostera E, Soto-Cerrato V. The prodigiosins, proapoptotic drugs with anticancer properties. Biochem Pharmacol. 2003;66(8):1447-52.

6. Pérez-Tomás $R$, Viñas $M$. New insights on the antitumoral properties of prodiginines. Curr Med Chem. 2010;17(21):2222-31.

7. Stankovic N, Senerovic L, llic-Tomic T, Vasiljevic B, Nikodinovic-Runic J. Properties and applications of undecylprodigiosin and other bacterial prodigiosins. Appl Microbiol Biotechnol. 2014;98(9):3841-58.

8. Darshan N, Manonmani HK. Prodigiosin and its potential applications. J Food Sci Technol. 2015;52(9):5393-407.

9. Suryawanshi RK, Patil CD, Borase HP, Salunke BK, Patil SV. Studies on production and biological potential of prodigiosin by Serratia marcescens. Appl Biochem Biotechnol. 2014;173(5):1209-21.

10. Danevčič T, Vezjak MB, Zorec M, Stopar D. Prodigiosin - A multifaceted Escherichia coli antimicrobial agent. PLoS One. 2016;11(9):9-15.

11. Castro AJ. Antimalarial activity of prodigiosin. Nature. 1967;213(5079):903-4.
12. Lazaro JE, Nitcheu J, Predicala RZ, Mangalindan GC, Nesslany F, Marzin $\mathrm{D}$, et al. Heptyl prodigiosin, a bacterial metabolite, is antimalarial in vivo and non-mutagenic in vitro. J Nat Toxins. 2002;11(4):367-77.

13. Azambuja P, Garcia ES, Ratcliffe NA. Gut microbiota and parasite transmission by insect vectors. Trends Parasitol. 2005;21(12):568-72.

14. McRary WL, Beaver EL, Noble ER. In vitro effects of prodigiosin and others antibiotics on Trypanosoma cruzi. Exp Parasitol. 1953;2(2):125-8.

15. Azambuja P, Feder D, Garcia ES. Isolation of Serratia marcescens in the midgut of Rhodnius prolixus: impact on the establishment of the parasite Trypanosoma cruzi in the vector. Exp Parasitol. 2004;107(1-2):89-96.

16. Genes C, Baquero E, Echeverri F, Maya JD, Triana O. Mitochondrial dysfunction in Trypanosoma cruzi: the role of Serratia marcescens prodigiosin in the alternative treatment of Chagas disease. Parasit Vectors. 2011;4:66.

17. Durán N, Justo GZ, Durán M, Brocchi M, Cordi L, Tasic L, et al. Advances in Chromobacterium violaceum and properties of violacein-Its main secondary metabolite: a review. Biotechnol Adv. 2016;34(5):1030-45

18. Rahul S, Chandrashekhar P, Hemant B, Bipinchandra S, Mouray E, Grellier P, et al. In vitro antiparasitic activity of microbial pigments and their combination with phytosynthesized metal nanoparticles. Parasitol Int. 2015;64(5):353-6.

19. Montaner B, Pérez-Tomás R. The prodigiosins: a new family of anticancer drugs. Curr Cancer Drug Targets. 2003;3(1):57-65.

20. Llagostera E, Soto-Cerrato V, Joshi R, Montaner B, Gimenez-Bonafé P, Pérez-Tomás R. High cytotoxic sensitivity of the human small cell lung doxorubicin-resistant carcinoma (GLC4/ADR) cell line to prodigiosin through apoptosis activation. Anticancer Drugs. 2005;16(4):393-9.

21. Dozie-Nwachukwu SO, Danyuo Y, Obayemi JD, Odusanya OS, Malatesta $\mathrm{K}$, Soboyejo WO. Extraction and encapsulation of prodigiosin in chitosan microspheres for targeted drug delivery. Mater Sci Eng C Mater Biol Appl. 2017;71:268-78.

22. Giri AV, Anandkumar N, Muthukumaran G, Pennathur G. A novel medium for the enhanced cell growth and production of prodigiosin from Serratia marcescens isolated from soil. BMC Microbiol. 2004;4:11.

23. Wang $X$, Tao J, Wei D, Shen $Y$, Tong W. Development of an adsorption procedure for the direct separation and purification of prodigiosin from culture broth. Biotechnol Appl Biochem. 2004;40(Pt 3):277-80.

24. Fonseca-Berzal C, Palmeiro-Roldán R, Escario JA, Torrado S, Arán VJ, Torrado-Santiago S, et al. Novel solid dispersions of benznidazole: preparation, dissolution profile and biological evaluation as alternative antichagasic drug delivery system. Exp Parasitol. 2015;149:84-91.

25. Vinuesa T, Herráez R, Oliver L, Elizondo E, Acarregui A, Esquisabel A, et al. Benznidazole nanoformulates: A chance to improve therapeutics for Chagas disease. Am J Trop Med Hyg. 2017;97(5):1469-76.

26. Ciccarelli AB, Frank FM, Puente V, Malchiodi EL, Batlle A, Lombardo ME. Antiparasitic effect of vitamin B12 on Trypanosoma cruzi. Antimicrob Agents Chemother. 2012;56(10):5315-20.

27. Suryawanshi RK, Patil CD, Koli SH, Hallsworth JE, Patil SV. Antimicrobial activity of prodigiosin is attributable to plasma-membrane damage. Nat Prod Res. 2017;31(5):572-7.

28. Benz R, Janko K, Boos W, Läuger P. Formation of large, ion-permeable membrane channels by the matrix protein (porin) of Escherichia coli. Biochim Biophys Acta. 1978;511(3):305-19.

29. Viñas M, Lorén JG, Guinea J. Particulate-bound pigment of Serratia marcescens and its association with cellular envelopes. Microbios Lett. 1983;24:19-26. 